\title{
BLANCHIMENT ACCÉLÉRÉ DES đEUFS NON FÉCONDÉS DETRUITE ARC-EN-CIEL (ONCORHYNCHUS MYKISS) SOUS L'EFFET DE SOLUTIONS SALINES EN VUE DE LEUR TRI MÉCANIQUE
}

\author{
S. MILLA, E. SAMBRONI et B. JALABERT
}

INRA-SCRIBE, IFR 140, Campus de Beaulieu, 35042 Rennes Cedex, France. Courriel : Sylvain.Milla@rennes.inra.fr

Reçu le 23 février 2006

Accepté le 15 décembre 2006

Received February 23, 2006

Accepted December 15, 2006

\section{RÉSUMÉ}

Le blanchiment des œufs non fécondés dans les pontes de salmonidés en incubation artificielle permet leur élimination par tri mécanique. Le déclenchement de ce phénomène normalement plus ou moins tardif durant l'incubation a pu être accéléré grâce à une brève exposition des œufs à une solution de sel de mer. Les modalités et les effets de cette exposition sur les taux de mortalité et de malformations des embryons ont été testés sur des pontes de truite arc-en-ciel à divers stades de développement (140, 190 et 240 degrés*jours). Le passage durant 1 à $20 \mathrm{~min}$ dans une solution saline de 30 à $120 \mathrm{~g} / \mathrm{l} \mathrm{a}$ permis d'augmenter significativement le taux de blanchiment des œufs non fécondés sans affecter celui des œufs fécondés. Toutefois, par un effet mécanique, la manipulation nécessaire au traitement a entraîné une augmentation significative de la mortalité embryonnaire pour une manipulation à 140 degrés*jours et du taux de malformations larvaires pour une manipulation à 190 degrés*jours. A 240 degrés*jours, la manipulation n'a pas affecté le développement embryonnaire mais a entraîné un retard de l'éclosion de quelques heures. Cette étude offre de nouvelles perspectives pour améliorer le tri mécanique des œufs fécondés et non fécondés au cours de l'incubation chez la truite arc-en-ciel.

Mots-clés: Oncorhynchus mykiss, incubation artificielle, chocs osmotiques, blanchiment de l'œuf.

\section{ACCELERATED WHITENING OF RAINBOW TROUT (ONCORHYNCHUS MYKISS) UNFERTILIZED EGGS UNDERTHE EFFECT OF SALINE SOLUTIONS FOR THEIR MECHANICAL SORTING}

\begin{abstract}
The whitening of unfertilized salmonid eggs during artificial incubation allows their elimination by mechanical sorting. This phenomenon that normally appears throughout the incubation period in rainbow trout could be accelerated by exposing shortly the eggs to marine salt solutions. The effects of various combinations of salt concentration and exposure time on further eggs whitening and embryos abnormalities rates were assayed at various developmental stages of rainbow trout eggs (140, 190 and 240 degree*days). The transfer during 1 to $20 \mathrm{~min}$ in a saline solution between 30 to $120 \mathrm{~g} / \mathrm{l}$ salinity increased significantly the whitening of unfertilized eggs without affecting that of fertilized ones. All treatments promoted a significant increase of embryonic mortality at 140 degree*days and of larval abnormalities at 190 degree*days, probably through a mechanical handling
\end{abstract}


effect. At 240 degree*days, handling did not affect the embryonic development but led to a hatching delay of a few hours. This study offers new prospects to improve mechanical sorting of unfertilized and fertilized rainbow trout eggs during incubation.

Key-words: Oncorhynchus mykiss, artificial incubation, osmotic shocks, egg whitening.

\section{INTRODUCTION}

Au cours de l'incubation artificielle des œufs de salmonidés, certains d'entre eux peuvent blanchir plus ou moins rapidement, dans des proportions variables selon la qualité des pontes. Ce phénomène résulte de la précipitation du vitellus au contact d'une entrée d'eau qui affecte des œufs abîmés lors des manipulations de prélèvement et d'insémination artificielle ou des œufs non fécondés. Dans ce dernier cas, le blanchiment peut intervenir tout au long de l'incubation, et parfois relativement tard. Dans tous les cas, ces œufs morts sont susceptibles de devenir le point de départ d'une prolifération fongique qui peut affecter l'ensemble des œufs en incubation et amplifier les mortalités. L'élimination des œufs blancs, par tri manuel ou mécanique, est toutefois délicate. En première partie d'incubation, jusqu'à environ 180 degrés*jours, les œufs embryonnés sont relativement sensibles aux manipulations (JENSEN J.O.T. et ALDERDICE D.F., 1983 ; JENSEN J.O.T. et ALDERDICE D.F., 1989), et des traitements chimiques sont généralement utilisés pour prévenir le développement de champignons. Au-delà de cette période les embryons sont beaucoup plus résistants, et le tri mécanique permet d'accélérer le blanchiment des œufs non fécondés grâce à des chocs modérés, et de procéder à leur élimination de manière automatisée. L'efficacité de cette technique paraît due au faible volume de l'espace périvitellin, ce qui rend la membrane vitelline particulièrement vulnérable aux déformations lorsque les manipulations sont effectuées hors de l'eau (JENSEN J.O.T. et ALDERDICE D.F., 1983). Cette méthode n'est toutefois pas parfaite, dans la mesure où tous les œufs non fécondés ne blanchissent pas nécessairement à la suite des chocs mécaniques, et où ceux-ci peuvent éventuellement affecter le développement des œufs embryonnés.

La présente étude a été réalisée pour évaluer la possibilité de provoquer le blanchiment des œufs non fécondés à différents stades de l'embryogenèse, par immersion de durée variable dans des solutions salines à diverses concentrations tout en minimisant les impacts négatifs éventuels sur les taux de survie et l'apparition de malformations.

\section{MATÉRIEL ET MÉTHODES}

Des truites arc-en-ciel de souche printanière (ovulation entre le $1^{\mathrm{er}}$ avril et le 15 juin), âgées de 2 ans, provenant de la SEMII (Station Expérimentale Mixte INRA-IFREMER, à Sizun) ont été transportées environ 6 semaines avant ovulation naturelle dans les installations expérimentales du laboratoire INRA-SCRIBE à Rennes et placées dans un circuit en eau recyclée à $10^{\circ} \mathrm{C}$, sous photopériode naturelle. Les femelles ont été soumises à un massage abdominal deux fois par semaine sous anesthésie au phenoxy-2-ethanol $(0,3 \%)$ afin de détecter l'ovulation. Les œufs de 4 femelles fraîchement ovulées ont été mélangés puis séparés en deux lots équivalents. L'un a été inséminé avec le mélange du sperme de 5 mâles: Sur $500 \mathrm{ml}$ d'ovules environ, lavés avec 2 I d'une solution saline de rinçage (Ovafish ${ }^{T M}$, IMV, France), est ajouté $200 \mu \mathrm{l}$ du pool de sperme puis 2 I d'une solution d'activation des spermatozoïdes (Actifish ${ }^{\mathrm{TM}}$, IMV, France). Après agitation très modérée puis incubation de $10 \mathrm{~min}$ au repos, les œufs sont transférés en incubateurs. L'autre lot a servi de témoin non fécondé (manipulations identiques sans adjonction de sperme). Les incubations des 2 groupes d'œufs ( 250 œufs par litre) se sont déroulées en circuit fermé filtré et thermorégulé à $10^{\circ} \mathrm{C}$ dans une eau saturée en oxygène renouvelée trois fois par minute environ. 
Après différentes durées d'incubation (140, 190, 240 degrés*jours, correspondant respectivement aux stades $21,24-25$, et $26 \mathrm{~b}$ de la table chronologique de développement embryonnaire de la truite (VERNIER J.M., 1969)), des lots de 100 œufs fécondés et 100 œufs non fécondés non blanchis ont été prélevés dans le circuit d'incubation puis plongés plus ou moins longtemps (1, 5 ou 20 minutes) dans des solutions de salinité variable $(0$, $5,30,60,90$ ou $120 \mathrm{~g} / \mathrm{l}$ de sel de Guérande dissout dans l'eau du circuit d'incubation), puis remis dans le circuit d'incubation (200 œufs par litre). Chaque traitement, effectué sur un unique lot de 100 œufs est donc caractérisé par la provenance des œufs, issus du groupe fécondé ou non, une durée d'incubation préalable, et un choc hypertonique de concentration et de durée variables. En outre, un lot témoin (en tétraplicat) d'œufs inséminés mais non manipulés a été utilisé pour évaluer l'impact du choc mécanique, imputable à la manipulation, sur le devenir des œufs fécondés.

L'effet des différents traitements a été évalué sur les variables suivantes :

- Le taux de blanchiment des œufs fécondés et non fécondés (TB) 24 heures après la manipulation.

- Le taux de malformations des alevins au moment de la résorption vitelline (rapport entre le nombre d'individus malformés et le nombre total d'individus à ce stade) et la typologie des malformations visibles extérieurement à ce stade (Voir résultats pour la description des malformations)

- Le taux de développement normal au moment de la résorption vitelline (rapport entre le nombre d'individus présentant un développement normal à ce stade et le nombre d'œufs embryonnés ayant subi la manipulation).

- La cinétique d'éclosion (pourcentage d'œufs éclos à différents instants après le début de l'éclosion).

Les valeurs des taux de blanchiment, de malformations et de développement normal ont été soumises à une analyse de variance non paramétrique de Kruskall-Wallis suivi par un test de Mann-whitney. La signification des différences entre cinétiques d'éclosion a été testée avec un test du Khi2. Le niveau de signification des différences observées a été fixé aux seuils de probabilité $p=0,05$ (significatif) et $p=0,01$ (hautement significatif).

\section{RÉSULTATS}

\section{Effet sur les taux de blanchiment des œufs fécondés et non fécondés}

Les manipulations effectuées dans cette étude ont entraîné le blanchiment d'une partie des œufs non fécondés entre 15 et 30 minutes après la fin de la manipulation. Sur les trois facteurs testés (durée d'incubation, durée de séjour dans l'eau salée et salinité), seule la salinité influe de façon significative sur le taux de blanchiment (TB) des œufs non fécondés $(p<0,01)$ (tableau I). Le TB est une fonction croissante de la salinité (Figure 1). On constate que l'effet du sel n'est significatif qu'à partir de $30 \mathrm{~g} / \mathrm{l}(\mathrm{p}<0,05)$. De plus, le TB est équivalent entre les salinités de 30 et $60 \mathrm{~g} / \mathrm{l}$. En revanche, le TB pour $90 \mathrm{~g} / \mathrm{l}$ est significativement supérieur au TB correspondant à la salinité de $60 \mathrm{~g} / \mathrm{l}$. Enfin, aucune différence significative n'est détectée entre $90 \mathrm{~g} / \mathrm{l}$ et $120 \mathrm{~g} / \mathrm{l}$, salinité pour laquelle le TB atteint une valeur maximale de $76 \%$ en moyenne. Au contraire, les traitements salins effectués dans notre étude n'influent pas significativement sur le TB des œufs fécondés (Tableau I) qui ne dépend significativement que de la durée d'incubation préalable $(p<0,01)$ (Figure 2). A 140 degrés*jours, le TB moyen (36\%) est significativement supérieur aux TB correspondant aux deux autres durées d'incubation testée $(p<0,01)$ où le TB est inférieur à $1 \%$. 


\section{Tableau I}

Niveaux de signification de l'ANOVA de Kruskall-Wallis sur l'effet de la durée d'incubation, de la salinité et de la durée de séjour dans l'eau salée sur les taux de blanchiment (TB), de malformations et de développement normal.

Table I

Kruskall-wallis ANOVA levels of significance on the effect of incubation duration, salinity and time exposure on whitening, abnormality and normal development rates.

\begin{tabular}{|l|l|l|l|}
\hline & $\begin{array}{l}\text { Durée } \\
\text { d'incubation }\end{array}$ & Salinité & $\begin{array}{l}\text { Durée de } \\
\text { séjour dans } \\
\text { l'eau salée }\end{array}$ \\
\hline TB des œufs non fécondés & 0.0666 & 0.0003 & 0.987 \\
\hline TB des œufs fécondés & $<10^{-4}$ & 0.9744 & 0.8323 \\
\hline Taux de malformations & $<10^{-4}$ & 0.7365 & 0.3552 \\
\hline - «Tire bouchon » & 0.0004 & 0.6588 & 0.0947 \\
\hline - «Morts/résorption » & $<10^{-4}$ & 0.9135 & 0.2161 \\
\hline - Déformations squelette & 0.022 & 0.5541 & 0.7399 \\
\hline $\begin{array}{l}\text { Taux de développement } \\
\text { normal }\end{array}$ & $<10^{-4}$ & 0.9207 & 0.6631 \\
\hline
\end{tabular}

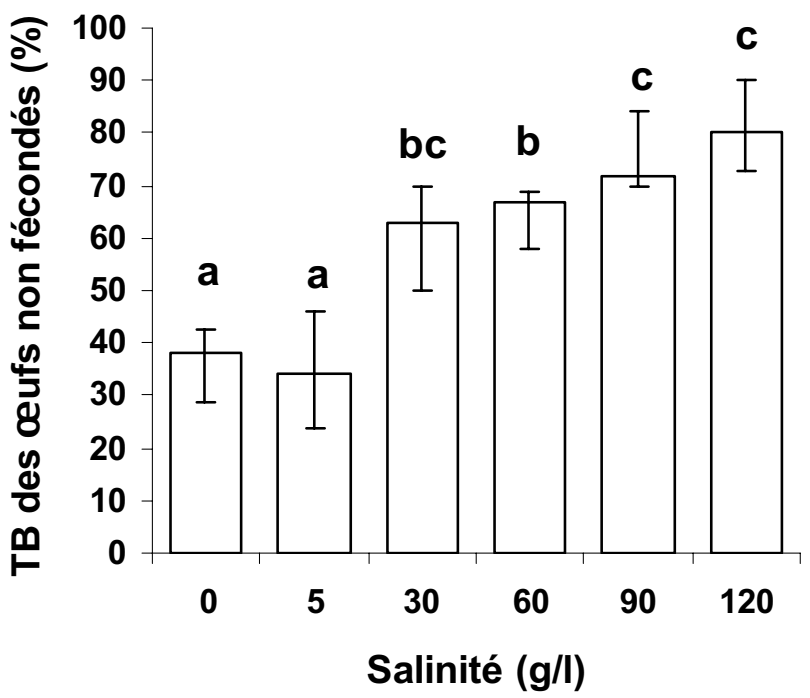

Figure 1

Effet de la salinité ( $\mathrm{g} / \mathrm{l})$ sur le taux de blanchiment (TB) des œufs non fécondés (médiane $\pm 75 / 25 \%$ quartile). Le TB est calculé 24 heures après la manipulation. Des lettres différentes indiquent des différences significatives entre les salinités $(p<0,05)$.

Figure 1

Effect of salinity ( $g / l)$ on the unfertilized egg whitening rate (TB) (median $\pm 75 / 25 \%$ quartile). TB is calculated $\mathbf{2 4}$ hours after handling. Different letters indicate significant differences between salinities $(p<0.05)$. 


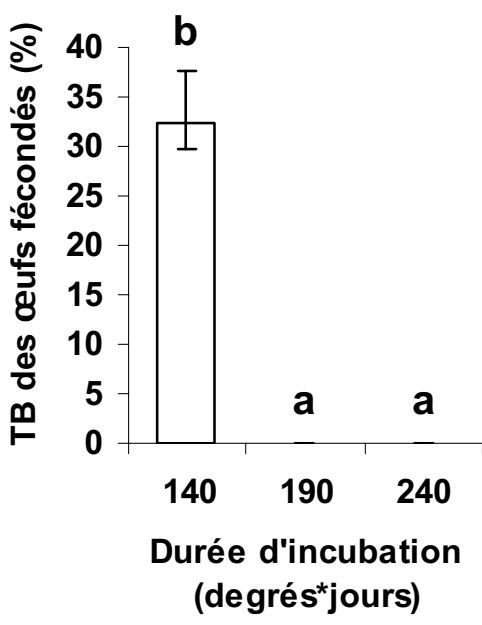

Figure 2

Effet de la durée d'incubation (140, 190 et 240 degrés*jours) sur le taux de blanchiment (TB) des œufs fécondés (médiane $\pm 75 / 25 \%$ quartile). Le TB est calculé 24 heures après la manipulation. Des lettres différentes indiquent des différences significatives entre les durées d'incubation $(p<0,05)$.

\section{Figure 2}

Effect of incubation stage $(140,190$ and 240 degree*days) on the fertilized egg whitening rate (TB) (median $\pm 75 / 25 \%$ quartile). TB is calculated 24 hours after handling. Different letters indicate significant differences between incubation durations $(p<0.05)$.

\section{Effet sur les taux de malformations}

Seule la durée d'incubation des œufs lors du traitement influe significativement sur le taux de malformations des juvéniles $(p<0,01)$ (tableau I). Le taux de malformations des alevins issus des œufs manipulés à 140 degrés*jours est significativement inférieur à celui du témoin non manipulé $(p<0,01)$ (Figure $3 A)$. Au contraire, une manipulation des œufs à 190 degrés*jours conduit à un taux de malformations significativement supérieur à celui du témoin non manipulé $(p<0,05)$. En revanche, le taux de malformations des

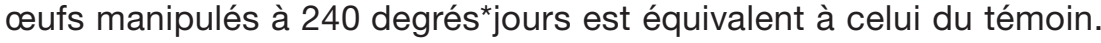

Sept types de malformation ont été répertoriés au terme de la résorption vitelline : "tire-bouchon " (le corps de l'animal est en forme de spirale), déformation du squelette (scoliose, lordose), atrophie céphalique, hydropisie (résorption incomplète de la vésicule vitelline), atrophie, atteinte de l'œil, siamois. A ces 7 catégories, nous rajoutons les "morts/résorption " qui sont les individus morts entre l'éclosion et la fin de la résorption vitelline. Trois anomalies externes de développement sont hautement majoritaires au terme de la résorption vitelline : les déformations du squelette (44\% des anomalies), les «tire-bouchon" $(29 \%)$ et les «morts/résorption» $(21 \%)$. Pour chacune, la fréquence d'apparition est uniquement liée à la durée d'incubation $(p<0,05)$ (tableau I). Les autres représentent chacune moins de $3 \%$ des anomalies. Seuls les alevins issus des œufs qui ont été manipulés à 190 degrés*jours ont un taux de déformation du squelette significativement supérieur à celui des autres lots manipulés et à celui du témoin $(p<0,05)$ (Figure 3B). Le taux de «tire-bouchon " des alevins issus des lots manipulés à 190 degrés*jours est significativement supérieur à celui des lots manipulés à 140 degrés*jours et à celui du témoin $(p<0,01)$ mais équivalent à celui des lots manipulés à 240 degrés*jours. Au contraire, les taux de «tire-bouchon » 
des lots manipulés à 140 et 240 degrés*jours sont équivalents au témoin (Figure $3 C$ ). Enfin, on observe une hausse significative du pourcentage de "morts/résorption " entre les lots manipulés à 140 degrés*jours et ceux à 190 degrés*jours (Figure 3D). Pour ces lots, ce taux est significativement inférieur au témoin $(p<0,01)$. Pour les lots manipulés à 240 degrés*jours, ce taux est équivalent au témoin et aux lots manipulés à 190 degrés*jours.

\section{A : Taux de malformations}

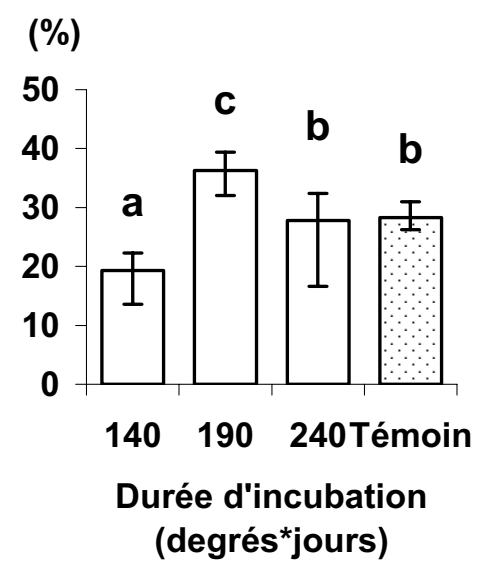

\section{B : Déformations du squelette}

(\%)

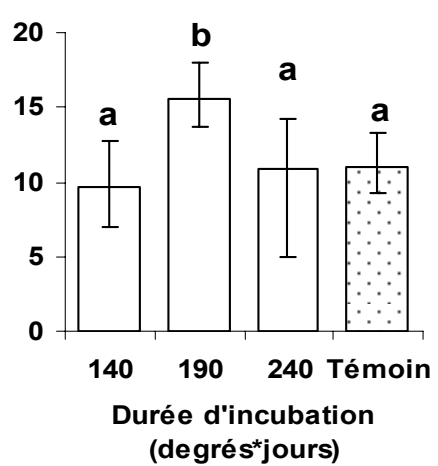

C : « Tire-bouchons »

(\%)

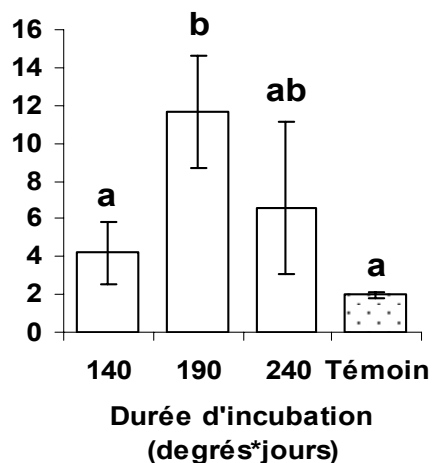

D : « Morts/résorption »

(\%)

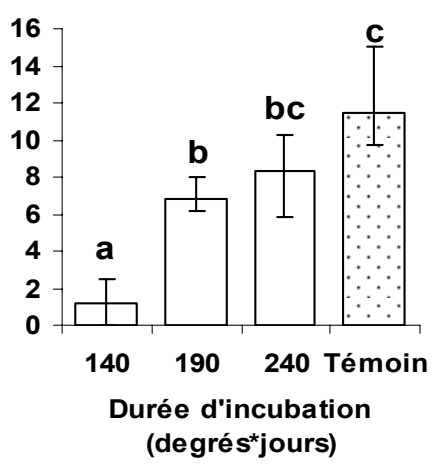

Figure 3

Effet de la durée d'incubation (140, 190 et 240 degrés*jours) sur le taux (\%) de malformations des alevins issus des œufs manipulés $(A)$, le taux de déformations du squelette (B), le taux de "tire-bouchon" (C) et le taux de mortalité entre l'éclosion et la résorption de la vésicule vitelline (D) (médiane $\pm 75 / 25 \%$ quartile). Le témoin correspond aux lots non manipulés. Des lettres différentes indiquent des différences significatives entre les durées d'incubation $(p<0,05)$.

\section{Figure 3}

Effect of incubation stage $(140,190$ and 240 degree*days) on juvenile fish malformation rate resulting from handled batches $(A)$, abnormality rate of the skeleton (B), "corkscrew" rate (C) and mortality rate between hatching and vitelline resorption (D) (median $\pm 75 / 25 \%$ quartile) The control corresponds to unhandled batches. Different letters indicate significant differences between incubation durations $(p<0.05)$. 


\section{Effet sur le taux de développement normal}

Seule la durée d'incubation lors de la manipulation a un effet sur le taux de développement normal au moment de la résorption vitelline $(p<0,01)$ (tableau $\mathrm{I}$ ). Les taux de développement normal des alevins issus des œufs manipulés à 140 et 190 degrés*jours (respectivement 43 et $63 \%$ ) sont significativement inférieurs au taux de développement normal du témoin non manipulé $(p<0,01$ et $p<0,05)$ (Figure 4). En revanche, la manipulation à 240 degrés*jours n'a pas affecté significativement le taux de développement normal.

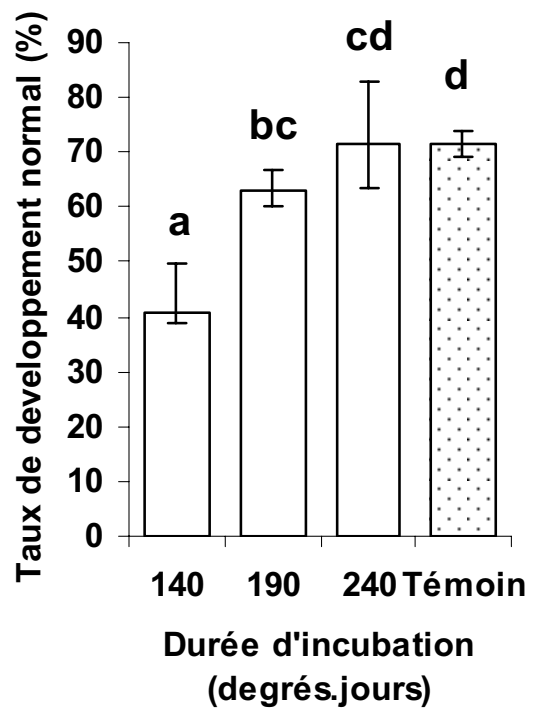

Figure 4

Effet de la durée d'incubation $(140,190$ et 240 degrés*jours) sur le taux de développement normal au moment de la résorption vitelline (médiane $\pm 75 / 25 \%$ quartile). Le taux de développement normal est défini comme le rapport entre le nombre d'individus présentant un développement normal et le nombre d'œufs inséminés initialement. Le témoin correspond aux lots non manipulés. Des lettres différentes indiquent des différences significatives entre les durées d'incubation $(p<0,05)$.

Figure 4

Effect of incubation stage $(140,190$ and 240 degree*days) on the normal development rate at vitelline resorption (median $\pm 75 / 25 \%$ quartile). The normal development rate is defined as the number of juvenile with normal development rate divided by the number of eggs initially inseminated. The control corresponds to unhandled batches. Different letters indicate significant differences between incubation durations $(p<0.05)$.

\section{Effet sur la cinétique d'éclosion}

Seule la durée d'incubation lors de la manipulation influe significativement sur la cinétique d'éclosion $(p<0,01)$ (Figure 5$)$. Le temps $t=0$ correspond à l'instant où le début de l'éclosion a été détecté. A cet instant, le taux d'éclosion avoisinait les $4 \%$ en moyenne et la première éclosion remontait à moins de 14 heures. La forme générale des courbes est sigmoïde avec une fréquence d'éclosion faible au départ, une augmentation progressive, une diminution de la fréquence des éclosions et enfin une tendance asymptotique vers le taux d'éclosion. Seul le profil d'éclosion des lots manipulés à 240 degrés*jours est significativement différent de celui du témoin non manipulé $(p<0,01)$. Au début de 
l'éclosion, les cinétiques de tous les lots manipulés sont confondues. C'est à partir de $t=6$ heures que le pourcentage d'éclos dans les lots manipulés à 240 degrés*jours est significativement inférieur au témoin $(p<0,05)$. Pour ces œufs, le retard final dans l'éclosion s'élève environ à 10 heures.

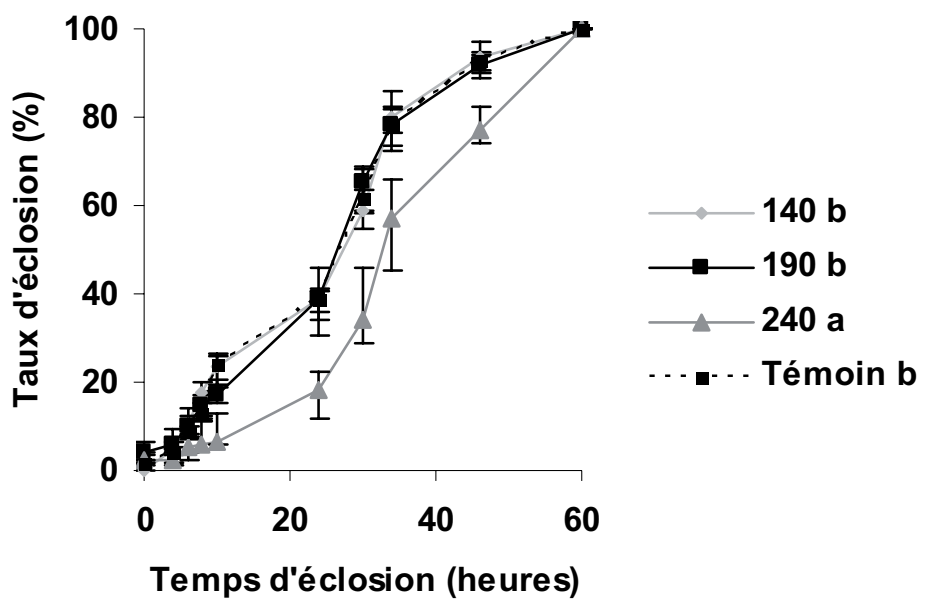

Figure 5

Évolution du taux d'éclosion (médiane $\pm 75 / 25 \%$ quartile) en fonction du temps, dans les lots issus des manipulations à 3 durées préalables d'incubation (140, 190 et 240 degrés*jours), et pour le témoin non manipulé. Des lettres différentes indiquent des différences significatives entre les durées d'incubation $(p<0,05)$.

Figure 5

Evolution of hatching rate (median $\pm 75 / 25 \%$ quartile) as a function of time, in

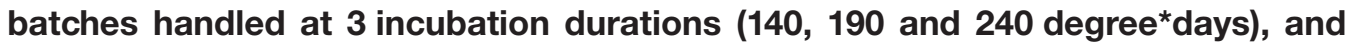
also for the unhandled control. Different letters indicate significant differences between incubation stages $(p<0.05)$.

\section{DISCUSSION}

La salinité est un facteur important pour le métabolisme, la survie et le développement des œufs de poissons. Certains paramètres directement liés à la salinité (pression osmotique, concentrations ioniques, oxygène...) ont en effet un impact sur les œufs et les embryons. L'effet d'un choc hypertonique transitoire relativement bref, tel que réalisé dans notre étude, ne semble pas avoir été étudié jusqu'à présent, alors qu'il semble permettre la discrimination entre des œufs non fécondés, destinés à mourir à plus ou moins brève échéance, et des embryons vivants dont le bon développement implique l'acquisition de facultés osmorégulatrices. En effet, l'utilisation du sel permet de provoquer efficacement le blanchiment de la majorité des œufs non fécondés sans affecter gravement les œufs fécondés. Dans cette étude, la manipulation comporte à la fois un stress mécanique modéré lié à la manipulation elle-même et un stress salin associé à l'immersion dans la solution salée. D'après nos résultats, le stress mécanique ne semble provoquer le blanchiment que d'environ $35 \%$ des œufs non fécondés, et le stress salin vient compléter significativement l'effet du stress mécanique. Mais une salinité faible $(5 \mathrm{~g} / \mathrm{l})$ est insuffisante pour provoquer une hausse significative du taux de blanchiment des œufs non fécondés. C'est à partir de $30 \mathrm{~g} / \mathrm{l}$ que le stress salin accentue ce taux de blanchiment et ce pourcentage augmente significativement avec la salinité, indépendamment du temps de séjour dans l'eau salée. Chez les œufs non fécondés de truite arc-en-ciel, une augmentation de l'absorption d'ions sodium et des échanges d'eau a été mise en évidence suite à une exposition à un milieu salin (KALMAN S.M., 1959). En outre, chez une espèce marine Pleuronectes 
platessa, une corrélation positive a été observée entre la salinité du milieu et l'osmolarité du vitellus (HOLLIDAY F.G.T. et JONES M.P., 1967). Les résultats de notre étude confirment que les œufs non fécondés de poissons ne régulent pas leur homéostasie ionique à la suite d'une forte augmentation de la salinité du milieu. II est probable que plus la salinité du milieu environnant est forte, plus l'œuf perd passivement de l'eau par phénomène d'osmose. Nous pouvons supposer que la perturbation des échanges aqueux et ioniques qui en découle provoquerait ensuite la mortalité de l'œuf. Cette mortalité marquée par un blanchiment de l'œuf est le signe d'un envahissement de l'œuf par l'eau provoquant ainsi une précipitation des globules vitellins (THEDINGA J.F et al., 2005).

En ce qui concerne le taux de blanchiment dans les lots d'œufs fécondés, il semble ne dépendre que de la durée préalable d'incubation, c'est-à-dire de leur stade de développement, et non de la salinité ni de la durée du traitement. Les œufs fécondés apparaissent sensibles à une manipulation trop précoce (140 degrés*jours) qui provoque un fort taux de blanchiment suite à la manipulation (36\% environ). Ce blanchiment semble imputable au stress mécanique de la manipulation plutôt qu'au stress osmotique puisqu'il ne dépend pas de la salinité du milieu. Chez la truite arc-en-ciel, une salinité de $8 \mathrm{~g} / \mathrm{l}$ semble constituer la limite supérieure de développement normal car une salinité de $12 \mathrm{~g} / \mathrm{l}$ affecte le taux de survie des œufs et la taille des alevins (SHEN A.C. et LEATHERLAND J.F., 1978 ; MORGAN J.D. et al., 1992). De plus, le transfert d'embryons de salmonidés d'eau douce en eau salée provoque une perturbation rapide des contenus en eau et en ions et leur mortalité après quelques dizaines d'heures (WEISBART, 1968 ; SHEN A.C. et LEATHERLAND J.F., 1978). Dans notre étude, nous pouvons supposer que les temps de séjour dans l'eau salée ont été trop courts pour affecter significativement le taux de blanchiment des œufs fécondés. En revanche, nos résultats sont cohérents avec les données sur l'évolution de la sensibilité des œufs de salmonidés aux chocs mécaniques au cours du développement embryonnaire. En effet, le stade de 140 degrés*jours se situe en phase finale de sensibilité critique aux chocs mécaniques (JENSEN J.O.T. et ALDERDICE D.F., 1983 ; JENSEN J.O.T. et ALDERDICE D.F., 1989). Par ailleurs, il est intéressant de constater que pour la manipulation à 140 degrés $^{*}$ jours, le taux de malformations au moment de la résorption vitelline est plus faible que celui du témoin non manipulé. Ce faible taux de malformations s'explique par un taux de " mortalité/résorption » très inférieur au témoin. Ce résultat suggère que, parmi le lot d'œufs blanchis suite à la manipulation (36\%), une forte proportion concerne des embryons qui seraient morts de toutes façons entre l'éclosion et la résorption vitelline même en l'absence de manipulations. Mais malgré ce faible taux de malformations au moment de la résorption vitelline, la forte mortalité intervenue suite à cette manipulation (36\%) explique un taux de développement normal plus faible que le témoin au moment de la résorption vitelline.

Au contraire, la manipulation à 190 degrés*jours n'a pas provoqué de mortalités mais a induit un surcroît de malformations. De fortes salinités sont responsables d'une hausse du pourcentage d'anomalies de développement chez divers téléostéens (DEPECHE J. et BILLARD R., 1994). Chez le saumon atlantique, une incubation à $12 \mathrm{~g} / \mathrm{l}$ provoque l'apparition de malformations larvaires (SAUNDERS J.W., 1966). Mais dans notre étude le taux de malformations apparaît indépendant de la salinité du traitement, et nous pouvons donc supposer que le choc osmotique était trop court pour provoquer un surcroît de malformations larvaires. A 190 degrés*jours, le développement embryonnaire est caractérisé par le développement de la queue et l'extension de la tête (VERNIER J.M., 1969). Nos résultats suggèrent que ces étapes du développement du squelette sont particulièrement vulnérables aux manipulations qui entraînent un taux de malformations supérieur au témoin non manipulé et par conséquent, un taux de développement normal inférieur au témoin.

Enfin, la manipulation à 240 degrés*jours n'a eu d'impact significatif ni sur la mortalité ni sur le taux de malformations. Ceci explique que le taux de développement 
normal des alevins issus des œufs manipulés à 240 degrés*jours est équivalent au témoin. Ce stade est donc le seul testé pour lequel le traitement ne semble pas avoir affecté le développement embryonnaire. En revanche, il a provoqué un allongement de la durée de la période d'éclosion.

\section{CONCLUSION}

Cette étude ouvre la voie à une nouvelle méthode utilisant un stress salin pour provoquer le blanchiment de la majorité des œufs non fécondés chez la truite arc-en-ciel sans affecter gravement les œufs embryonnés. Le stress mécanique de la manipulation s'est toutefois avéré susceptible d'affecter le développement embryonnaire aux deux stades testés les plus précoces (140 et 190 degrés*jours). Il devra être fortement minimisé pour envisager une application éventuelle. C'est après 240 degrés*jours d'incubation que la méthode du stress salin paraît aisément applicable sans inconvénients majeurs. A ce stade, un traitement efficace consisterait à plonger les œufs incubés dans un bain d'eau salée à 90 ou $120 \mathrm{~g} / \mathrm{l}$ pendant une minute, puis à les transvaser immédiatement dans le circuit d'incubation, et à attendre environ 1 heure avant d'éliminer mécaniquement les œufs blancs.

\section{REMERCIEMENTS}

Les auteurs remercient chaleureusement C. DURET pour sa collaboration technique.

\section{BIBLIOGRAPHIE}

DEPECHE J. and BILLARD R., 1994. Embryology in fish a review. Société Française d'Ichtyologie, Paris, $118 \mathrm{p}$.

HOLLIDAY F.G.T., JONES M.P., 1967. Some effects of salinity on the developing eggs and larvae of the plaice (Pleuronectes platessa). Marine Biol. Assoc. U.K., 47, 39-48.

JENSEN J.O.T., ALDERDICE D.F., 1983. Changes in mechanical shock sensitivity of coho salmon (Oncorhynchus kisutch) eggs during incubation. Aquaculture, 32, 303-312.

JENSEN J.O.T., ALDERDICE D.F., 1989. Comparison of mechanical shock sensitivity of eggs of five Pacific salmon (Oncorhynchus) species and steelhead trout (Salmo gairdneri). Aquaculture, 78, 163-181.

KALMAN S.M., 1959. Sodium and water exchange in the trout egg. Cell. Physiol., 54, 155162.

MORGAN J.D., JENSEN J.O.T., IWAMA G.K., 1992. Effects of salinity on aerobic metabolism and development of eggs and alevins of steelhead trout (Oncorhynchus mykiss) and fall chinook salmon (Oncorhynchus tshawytscha). Can. J. Zool., 70, 1341-1346.

SAUNDERS J.W., 1966. Estuarine spawning of Atlantic Salmon. J. Fish. Res. Bd., 23, 1803-1804.

SHEN A.C., LEATHERLAND J.F., 1978. Effect of ambient salinity on ionic and osmotic regulation of eggs, larvae, and alevins of rainbow trout (Salmo gairdneri). Can. J. Zool., 56, 571-577.

THEDINGA J.F, CARLS M.G., MASELCO J.M., HEINTZ R.A., RICE S.D., 2005. Resistance of naturally spawned pink salmon eggs to mechanical shock. Alaska Fishery Research bulletin, 11, 37-43. 
VERNIER J.M., 1969. Table chronologique du développement embryonnaire de la truite arcen-ciel, Salmo gairdneri Rich. 1836. Annales d'embryologie et de morphogenèse, 2, 495-520.

WEISBART, M., 1968. Osmotic and ionic regulation in embryos, alevins, and fry of the five species of Pacific salmon. Can. J. Zool., 46, 385-397. 